\title{
Hysteresis Characterization Using Charge-Feedback Control for a LIPCA Device
}

\author{
James Beck ${ }^{a}$, Maciej Noras ${ }^{b}$, Jerzy Kieres $^{b}$, John E. Speich $^{a}$, \\ Karla M. Mossi ${ }^{a}$ and Kam K. Leang ${ }^{a}$ \\ ${ }^{a}$ Virginia Commonwealth University, Department of Mechanical Engineering, \\ 601 West Main Street, PO BOX 843015, Richmond, VA, 23284-3015, USA; \\ ${ }^{b}$ Trek Incorporated, 11601 Maple Ridge Road, Medina, NY 14104, USA
}

\begin{abstract}
In this paper, we study the no-load behavior of a lightweight piezo-composite curved actuator (LIPCA) subjected to voltage and charge control. First, we examine the effect of hysteresis and creep when the actuator is voltage controlled at a slow scan speed. The experimental results show that creep increases the displacement hysteresis by over $25 \%$ when scanning at $1 / 60 \mathrm{~Hz}$. Afterwards, we discuss the design and implementation of a charge-feedback circuit to control the displacement of the actuator. The hysteresis curves between voltage- and charge-control modes are compared for the scan frequencies of 1 and $5 \mathrm{~Hz}$. The results show that charge control (compared to voltage control) of a LIPCA device exhibits significantly less hysteresis - over $80 \%$ less.
\end{abstract}

Keywords: Pre-stressed piezoactuators, charge-feedback control, hysteresis, and creep.

\section{INTRODUCTION AND MOTIVATION}

In this paper, we study the behavior of a pre-stressed composite piezoelectric actuator ${ }^{1,2}$ subjected to open-loop voltage and charge-feedback control. In particular, we focus on the no-load displacement behavior of a lightweight piezo-composite curved actuator (LIPCA)*. First, we examine the effect of hysteresis and creep at a slow scan speed. Then, we compare how voltage and charge-feedback control affects the displacement of a LIPCA device.

LIPCA devices are composite actuators manufactured by sandwiching (at high temperatures) a soft-piezoceramic layer, type PZT-5A, between glass epoxy and carbon epoxy layers ${ }^{3}$ (e.g. , see Fig. 1(a)). These devices produce moderate to high strains, ${ }^{4}$ and because they can generate large movements in addition to high forces, LIPCA-type actuators are attractive, for example, as control surfaces in aerospace ${ }^{5}$ applications and as active diaphragms ${ }^{2}$ in synthetic jets for flow control. Although they can achieve large movements, a significant hysteresis effect - much as 15-20 percent of the total displacement range - has been observed when they are voltage controlled. ${ }^{6}$ In general, it is more convenient (compared to charge control) ${ }^{7}$ to control the displacement of a piezoactuator by varying the voltage across its electrodes, but voltage control has several drawbacks. For example, the relationship between applied voltage and the piezoactuator's displacement is very hysteretic. Hysteresis presents a challenge when piezos are used for high-precision positioning applications, such as in scanning probe microscopes - piezoactuators are used to move a probe-tip relative to a sample surface. ${ }^{8}$ Additionally, when piezoactuators are used as control surfaces on an aircraft, ${ }^{5}$ hysteresis can limit performance by reducing repeatability, and in some cases, it can destabilize the controlled system. ${ }^{9}$ On the other hand, it has been shown that charge control significantly reduces the hysteresis in tube-shaped- ${ }^{10}$ stacked-,${ }^{11,12}$ and plate-type piezoactuators. ${ }^{13}$ Unfortunately, limited studies exist on the topic of charge control for LIPCA-type devices. Therefore, a contribution of this paper is studying the effect of charge control on a LIPCA-type device. The behavior of the actuator under charge control is compared to voltage control. Additionally, we examine the effect of hysteresis and creep at a slow scan speed.

This paper is organized as follows: In the next section we describe the experimental LIPCA system. In Section 3 we discuss the effects of hysteresis and creep at a slow scan speed. Then, we describe in Section 4 the effect of voltage and charge control on a LIPCA device, followed by concluding remarks and acknowledgements.

Corresponding author's email: kkleang@vcu.edu, kmmossi@vcu.edu, Maciej.Noras@trekinc.com

*Manufactured by Konkuk University, Seoul Korea. 


\section{THE EXPERIMENTAL SYSTEM}

In this section, we describe in detail the experimental system, which consists of a LIPCA device, a laser displacement sensor, piezoelectric device amplifiers, and a computer and data acquisition system. We begin the discussion by describing the LIPCA device.

\subsection{The LIPCA Device}

The experimental LIPCA device is made of a piezoelectric ceramic layer (lead zirconate titanate or PZT) sandwiched between two layers of mixed fiberglass and carbon composite as shown in the Fig. 1(a). In particular, the actuator is composed of a glass/epoxy top layer of $100 \mathrm{~mm} \times 24.0 \mathrm{~mm} \times 0.89 \mathrm{~mm}$, a unidirectional carbon/epoxy layer of $71 \mathrm{~mm} \times 22 \mathrm{~mm} \times 0.1 \mathrm{~mm}$, another glass/epoxy layer, a PZT-5A layer of $72.3 \mathrm{~mm} \times 23.0$ $m m \times 0.25 \mathrm{~mm}$, and another glass/epoxy layer. Electrodes are built into the layers with copper strips so that direct contact with the PZT-5A layer is possible. The device is pre-stressed due to the difference in coefficients of thermal expansion of the layers since the composite is processed at high temperature $\left(300^{\circ} \mathrm{C}\right)$. This process has demonstrated improved performance. ${ }^{14}$ The nominal geometry of the actuator is shown in Fig. 1(b), where the difference in coefficients of thermal expansion of the layers causes it to exhibit a curved shape. A potential difference (voltage) applied across the electrodes of a LIPCA device causes it to displace (e.g., in the $y$-direction as illustrated in Figs. 1(c) and (d)) - positive voltage causes a deformation that decreases the radius of curvature of the device, and negative voltage "flattens" it (e.g., see Fig. 1(d)). Mossi et al. ${ }^{2}$ and Haris et al. ${ }^{3}$ describe experimental and numerical studies of the performance for LIPCA-type devices.

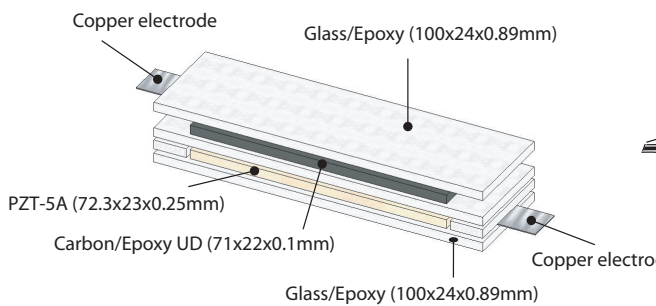

(a)

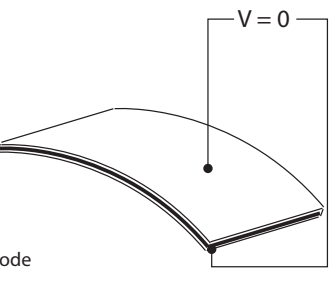

(b)

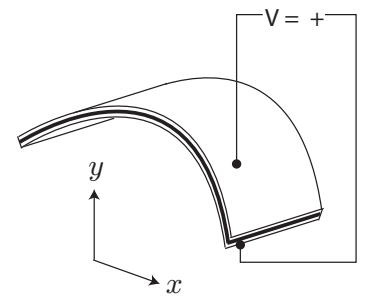

(c)

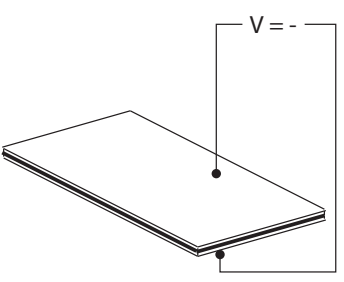

(d)

Figure 1. (a) A LIPCA device. (b) (Curved) nominal shape when applied voltage is zero. (c) Increase curvature when voltage is positive. (d) The actuator "flattens" when the applied voltage is negative.

\subsection{The Experimental Setup}

The experimental piezo system is shown in Fig. 2. The LIPCA device described in the preceding section is fixed, but free to rotate, at location $A$, and it can translate along the $x$-direction at location $B$. A laserdisplacement sensor, NAIS Model ANR12511 (with gain of $2.0106 \mathrm{~mm} / \mathrm{V}$ ), measures the out-of-plane motion (in the $y$-direction) of the actuator. The sensor has a measurable range of $\pm 10 \mathrm{~mm}( \pm 0.394 \mathrm{inch})$ and resolution of $1 \mu \mathrm{m}$ at $10 \mathrm{~Hz}$; the resolution reduces to $3.5 \mu \mathrm{m}$ at $100 \mathrm{~Hz}$. Additionally, the sensor has linearity of $0.2 \%$ of full scale and the output signal $(\tilde{y})$ is amplified and low-pass filtered (cut-off frequency at $5 \mathrm{kHz}$ ) before it is measured. A computer (PC) equipped with a 12-bit National Instruments LabPC+ data acquisition (DAQ) board measures the sensor signal $\hat{y}$ (see Fig. 2). The DAQ board also provides a command voltage (either $u_{v}$ for voltage control, or $u_{c}$ for charge control) to the piezo amplifiers to control its displacement. In this study, the experimental LIPCA device is actuated by: (1) controlling the voltage $u_{v}$ across the electrodes (voltage-control mode) and (2) controlling the charge $u_{c}$ delivered to the device (charge-control mode). Next, we briefly describe the voltage and charge-feedback-based piezoelectric device amplifiers.

\subsection{The Piezoelectric Device Amplifiers}

In the experiments, we use a Trek Inc. high-voltage amplifier (Model PZD-2000) with a gain of $60(V / V)$ to control the voltage applied to the actuator. A reference voltage $u_{v}$ from the PC/DAQ system is low-pass filtered 


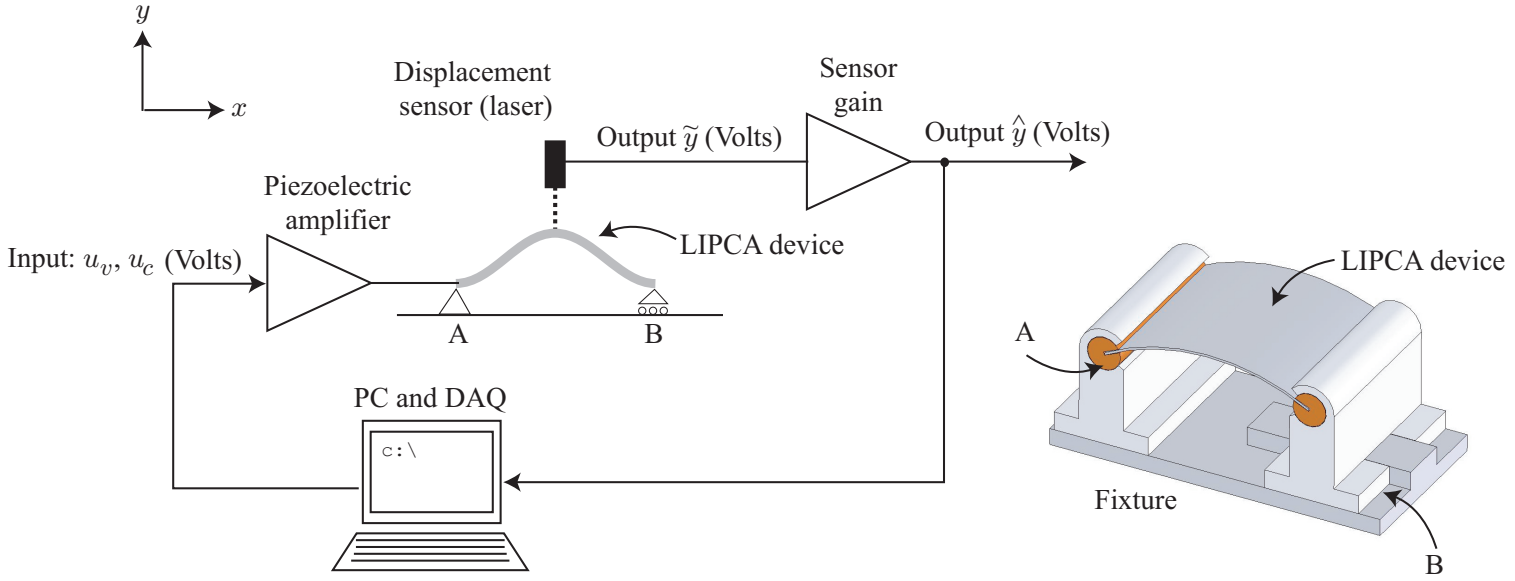

Figure 2. The experimental piezo system. A LIPCA device is fixed at location $A$ and free to translate along the $x$ direction at $B$. A computer (PC) and data acquisition system (DAQ) provide either a reference voltage $u_{v}$ or charge $u_{c}$ to the piezo amplifier for actuating the LIPCA device. The out-of-plane displacement $y$ of the piezo is measured by a laser which outputs a signal $\tilde{y}$ in volts. The sensor output $\tilde{y}$ is further amplified and filtered before the signal $\hat{y}$ is measured by the PC/DAQ system.

(at a cut-off frequency of $5 \mathrm{kHz}$ ) before it is applied as an input to the high-voltage amplifier. The filter reduces the effects of high-frequency noise.

Charge control is another method to activate a piezoactuator. ${ }^{15}$ Researchers have exploited this method for various positioning applications. ${ }^{10-12,16}$ It has been demonstrated that charge control reduces the hysteresis effect in piezoelectric materials. In this study, we apply charge control to the experimental LIPCA device and study its effect. Figure 3 shows the design for a feedback-based charge amplifier (based on the Trek PZD-700 high-voltage amplifier) used to control the LIPCA device. In the design, a feedback circuit maintains control of a reference input charge, $u_{c}$ (in volts). A charge monitor, based on the AD210 precision isolation amplifier, detects dynamic changes of charge delivered to a piezoelectric load and the information is processed by an amplifier (Analog Devices OP42FZ) to minimize the error between the desired input charge and actual charge on the electrodes of the actuator. An auxiliary voltage feedback loop helps minimize DC offset effects.

\section{EXPERIMENTAL INVESTIGATION: HYSTERESIS AND CREEP}

In voltage-control mode piezoelectric materials exhibit, in addition to hysteresis behavior, creep (drift) effect when they are operated over long periods of time. ${ }^{17,18}$ For example, the measured step response of the LIPCA device in Fig. 6(b1) shows drifting of the actuator's displacement over a 10-minute period. In this section, we examine how creep affects the displacement hysteresis in the experimental LIPCA device. We begin with a brief discussion on minimizing dynamics effect such that induced structural vibrations do not affect the measurement of displacement hysteresis.

\subsection{Minimizing Vibration Effect}

To avoid the effect of induced structural vibrations (i.e., oscillations), we applied inputs with sufficiently low frequency components to the LIPCA device to avoid exciting its resonant modes. We determined the inputfrequency range by measuring and evaluating the vibrational dynamics (in voltage-control mode) of the piezoactuator in the $y$-direction using a dynamic signal analyzer (DSA, Hewlett Packard Model 35670A). The DSA applied a sinusoidal input voltage $u_{v}$ (Volts) to actuate the piezo in the $y$-direction and the laser sensor measured the resulting displacement (in Volts). The input and output information was used to construct the frequency response. Additionally, we actuated the LIPCA device over small displacement ranges $( \pm 40 \mu \mathrm{m})$ where hysteresis is negligible, and Fig. 4 shows the magnitude and phase versus frequency plots. 


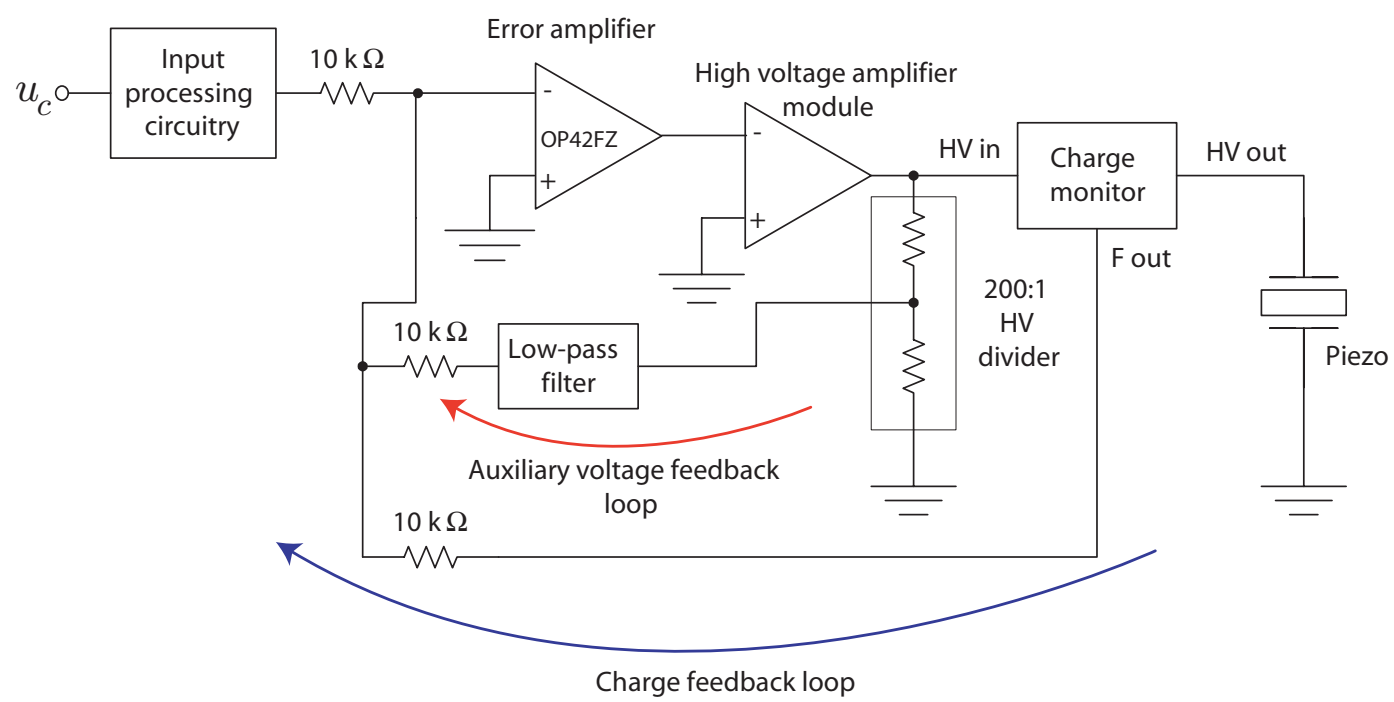

Figure 3. A charge-feedback amplifier design.
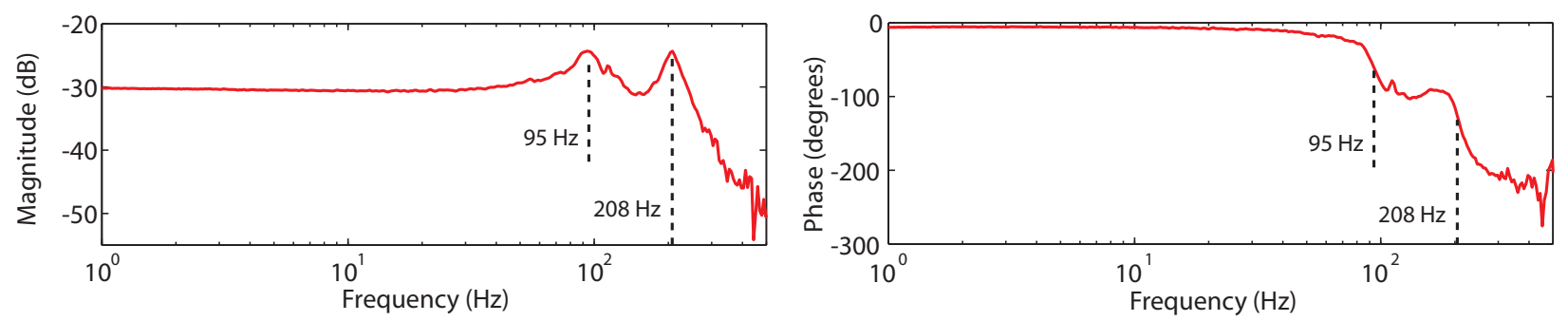

Figure 4. The frequency response (magnitude and phase versus frequency) for the LIPCA device under voltage-control mode. The frequency response was obtained over small displacement range $( \pm 40 \mu \mathrm{m})$ where hysteresis is negligible.

Based on the results in Fig. 4, we observe that the experimental LIPCA device has two dominant resonant peaks, one at $95 \mathrm{~Hz}$ and one at $208 \mathrm{~Hz}$ (see Fig. 4). To avoid exciting the effect of the two peaks, we chose for all experiments in this paper scan frequencies equal to and below $5 \mathrm{~Hz}$. The frequency range is less than roughly $5 \%$ of the lowest resonant peak at $95 \mathrm{~Hz}$. In particular, we considered low frequency triangle and sinusoidal input signals.

\subsection{Compensating for Creep (Drift) Effect}

To isolate the hysteresis behavior from the creep (drift) effect, we used an inversion-based approach ${ }^{19,20}$ to find a creep-free output response. Then we quantify the contribution that creep has on the displacement hysteresis for the LIPCA device.

For a given desired displacement, the inversion-based approach finds an input that compensates for creep behavior (see Fig. 6(a1)). At low-scan speeds $(<1 \mathrm{~Hz})$, we used this approach to generate the creep-compensated hysteresis curves. The procedures are as follows:

Step 1: Model the Creep Effect - We modeled the creep effect, which occurs both in the mechanical and electrical domains, in the LIPCA device using a series connection of dampers $\left(c_{i}\right)$ and springs $\left(k_{i}\right)$ as illustrated 
in Fig. 6(a2). ${ }^{21}$ The following transfer function describes the creep model:

$$
G_{c}(s) \triangleq \frac{\hat{y}(s)}{u_{v}(s)}=\frac{1}{k_{0}}+\sum_{i=1}^{n} \frac{1}{s c_{i}+k_{i}}
$$

where $\hat{y}(s)$ is the measured displacement of the piezo (in the Laplace domain) and $u_{v}(s)$ is the applied input voltage. In the above equation, $k_{0}$ models the elastic behavior at low frequencies and the creep behavior is captured by selecting an appropriate model order $n$, or equivalently, the number of damper-spring elements. We found the parameters $\left(k_{0}, k_{i}\right.$, and $\left.c_{i}\right)$ in Eq. (1) by curve fitting (using a least-squares technique) the step response of the piezo measured over a period of 10 minutes (see Fig. 6(b1)). ${ }^{22}$ An input of magnitude $u_{v}=500 \mathrm{mV}$ ( $\leq 10 \%$ of full scale) was applied to the LIPCA device to generate the step response. The small input was chosen to avoid hysteresis effect which dominates over large displacement ranges. From the measured (displacement) response, we obtained the following second-order $(n=2)$ creep model (units of $V / V)$ :

$$
G_{c}(s)=\frac{0.3266 s^{2}+0.0709 s+0.000324}{s^{2}+0.1850 s+0.000724}
$$

The error between the model output and measured creep response is shown in Fig. 6(b2). The maximum and minimum errors are $+2.24 \mu \mathrm{m}$ and $-2.17 \mu \mathrm{m}$, respectively. In comparison, the resolution of the displacement sensor is $1 \mu \mathrm{m}$ at $10 \mathrm{~Hz}$ and $3.5 \mu \mathrm{m}$ at $100 \mathrm{~Hz}$.

Step 2: Find Input to Compensate for Creep - We determined an input - one that compensates for creep - using an inversion-based approach. ${ }^{19,20}$ We applied the method and Figs. 6(c1) and (c2) show the results of the approach for small $( \pm 44 \mu \mathrm{m})$ and large $( \pm 355 \mu \mathrm{m})$ displacements, respectively. The desired output is a triangle signal with an amplitude that decays linearity to a negative offset value. Without compensation, the final value creeps by $12 \mu \mathrm{m}$ for the small range case and by $133 \mu \mathrm{m}$ for the large range case. Therefore, when the LIPCA device is operated over long periods of time drift is significant; however, our results show that it can be accounted.

Remark: In charge-control mode, we measured significantly less creep as shown in Fig. 6(b1). Compared to voltage-control mode, the creep effect is smaller because the charge amplifier is believed to actively control the amount of charge on the electrodes of the LIPCA device.

\subsection{Quantifying the Displacement Hysteresis}

We determined the displacement hysteresis for all experiments from the input and output signals as follows: First, we plot the input versus output variables ( $y$ vs. $u$ ) to obtain the hysteresis curve (e.g. , see Fig. 5). At steady-state, each hysteresis loop in the input-output plane is partitioned into an ascending branch $\mathcal{B}_{A}$ and descending branch $\mathcal{B}_{D}$ as shown in Fig. 5. Then, we calculate the displacement hysteresis, $D_{h}(u)$, by taking the difference between the measured output on the ascending and descending branches. $D_{h}(u)$ is a function of the input $u$.

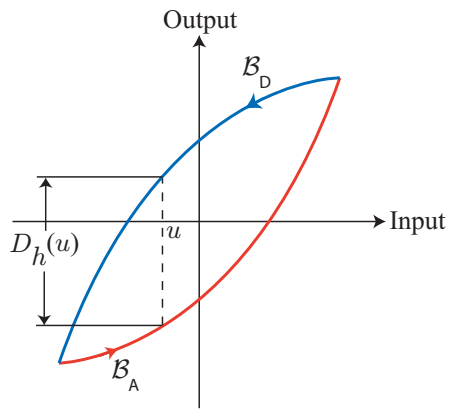

Figure 5. Displacement hysteresis.

\subsection{Experimental Results: Hysteresis and Creep Behavior}

We conducted experiments to study the displacement behavior for the LIPCA device at a slow scan speed. A $3 \mathrm{~V}$ triangular input signal with frequency $1 / 60 \mathrm{~Hz}$ was applied to the high-voltage amplifier. With a gain of $60(V / V)$, the high-voltage amplifier delivered $180 \mathrm{~V}$ to the actuator. The desired range for the actuator in this experiment was $\pm 450 \mu \mathrm{m}$. The solid line in Fig. 6(d1) is the hysteresis curve without creep compensation. Figure 6(d2) shows the measured displacement hysteresis $D_{h}$ versus the applied voltage $u_{v}$ across the electrodes of the piezoactuator. Without creep compensation, the maximum displacement hysteresis was $239.71 \mu \mathrm{m}$, or $26.75 \%$ of the total displacement range $(2 \times 450 \mu \mathrm{m})$. By accounting for creep, the displacement hysteresis is less. In fact, experimental results show that over the same displacement range, creep increases the displacement hysteresis by over $25 \%$ as shown in Figs. $6(\mathrm{~d} 1)$ and $(\mathrm{d} 2)$ - the maximum displacement hysteresis $\left(D_{h}\right)$ with creep compensation is $189.23 \mu \mathrm{m}$ as opposed to $239.71 \mu \mathrm{m}$ without creep compensation. Therefore, creep effect is significant at low scan speed and it contributes significantly to the displacement hysteresis. 
(a1)

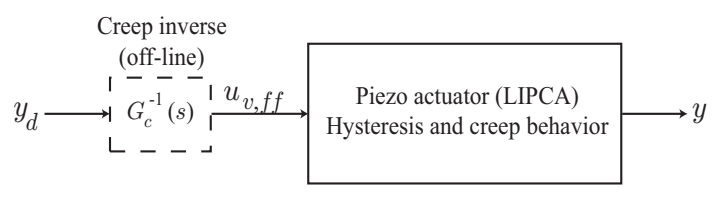

(b1)

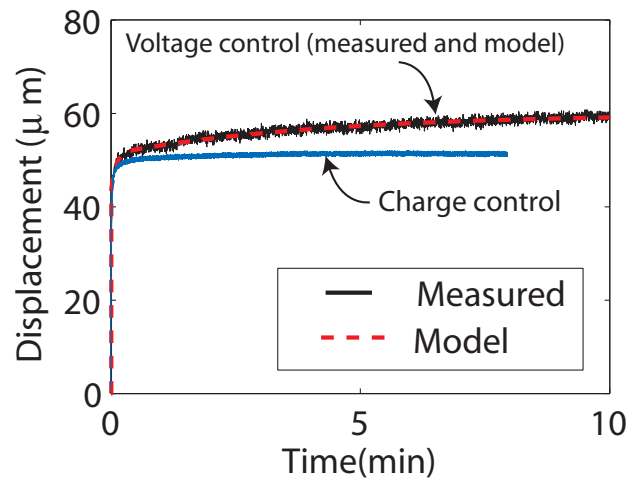

(c1)

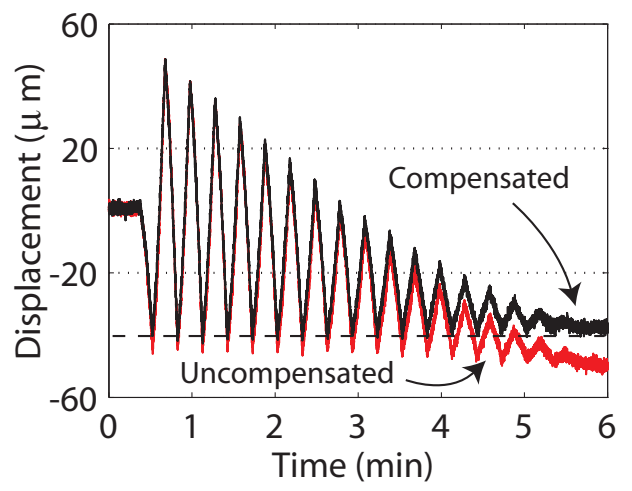

(d1)

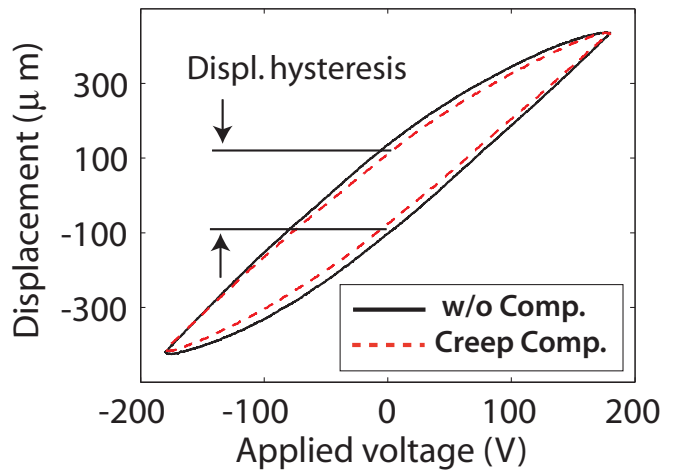

(a2)

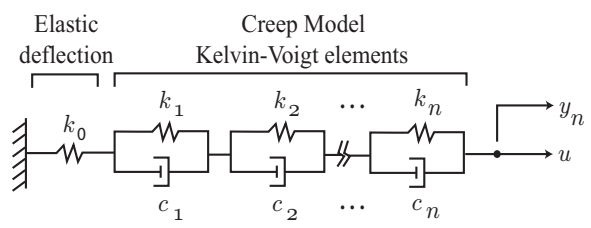

(b2)

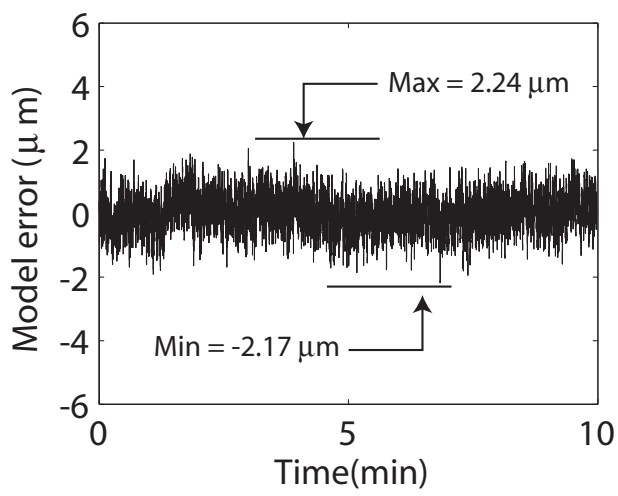

(c2)

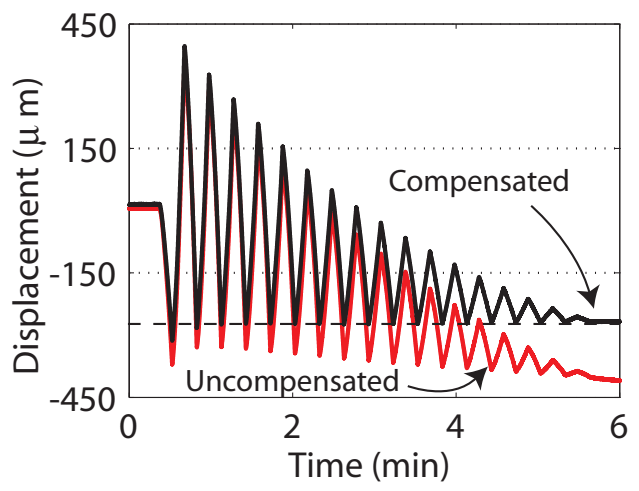

$(d 2)$

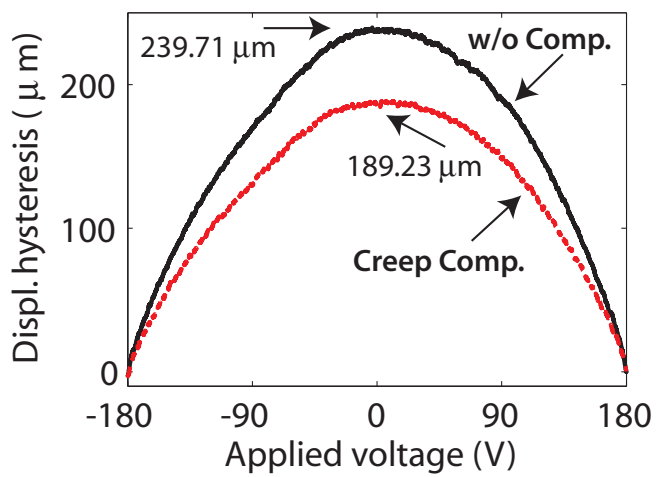

Figure 6. Hysteresis effect with and without creep compensation. (a1) Feedforward approach to compensate for creep. ${ }^{19,20}$ (a2) The lumped-parameter creep model consisting of spring and damper elements. (b1) Step response: solid line represents measured response with creep behavior; dashed line represents creep model. (b2) Model error vs. time. (c1) Measured displacement over small range with and without creep compensation. (c2) Measured displacement over large range with and without creep compensation. (d1) Hysteresis curves acquired at 1/60 $\mathrm{Hz}$, with and without creep compensation. (d2) Displacement hysteresis, $D_{h}(u)$, with and without creep compensation. 


\section{EXPERIMENTAL INVESTIGATION: VOLTAGE VS. CHARGE CONTROL}

We conducted eperiments to study and compare the effects of open-loop voltage and charge-feedback control on the displacement hysteresis for the experimental LIPCA device. We begin by describing the input signals considered in the experiments.

\subsection{Experimental Procedures}

In the experiments, we chose sinusoidal inputs with frequencies of $1 \mathrm{~Hz}$ and $5 \mathrm{~Hz}$, which are significantly lower than the lowest dominant resonant frequency at $95 \mathrm{~Hz}$ as indicated in Fig. 4. Additionally, sine inputs were chosen over triangle inputs because sinusoids do not contain high frequency components at the turn-around points, which can induce oscillations. At each frequency, hysteresis curves for three different displacement ranges were obtained: (1) small range - $70 \mu m( \pm 35 \mu m)$, (2) medium range $-250 \mu m( \pm 125 \mu m)$, and (3) large range $-700 \mu \mathrm{m}( \pm 350 \mu \mathrm{m})$. For convenience we considered as the input variable the desired displacement when plotting the hysteresis curve. That is, the hysteresis curves are obtained by plotting the measured displacement (output) versus the desired displacement (input). The method described in Section 3.3 was used to calculate the displacement hysteresis, $D_{h}$. We note that if the measured displacement has no distortion (due to hysteresis), the relationship between the measured displacement and the desired displacement is linear.

\subsection{Experimental Results: Voltage vs. Charge Control}

The experimental results are presented in Figs. 7 and 8 for $1 \mathrm{~Hz}$ and $5 \mathrm{~Hz}$ scan frequencies, respectively. Both figures show the hysteresis curves and measured displacement hysteresis for the three different displacement ranges: $70 \mu \mathrm{m}, 250 \mu \mathrm{m}$, and $700 \mu \mathrm{m}$. From the results, we immediately notice that charge control for a LIPCA device produces significantly less hysteresis. Additionally, the following observations were noted:

In both voltage and charge control, for the small displacement range $(70 \mu m)$ the resulting displacement hysteresis is relatively small. Figures 7(a1) and (a2), and Figs. 8(a1) and (a2) show the results for $1 \mathrm{~Hz}$ and $5 \mathrm{~Hz}$, respectively. Noise effects did not allow accurate measurements, however, an estimate shows that the displacement hysteresis is on the order of $5-7 \%$ (and possibly smaller) when the range is $70 \mu m( \pm 35 \mu m)$.

The experimental results clearly show that as the displacement range increases, the displacement hysteresis under voltage control increases linearly with the range. A plot of displacement hysteresis versus the range is shown in Fig. 8(d) and at $1 \mathrm{~Hz}$, the trend is approximately $23.7 \mu \mathrm{m}$ per $100 \mu \mathrm{m}$ of range. At $5 \mathrm{~Hz}$, however, the rate is slightly over half, at $13.6 \mu \mathrm{m}$ per $100 \mu \mathrm{m}$ of range. Interestingly, the linearity holds for charge control: at $1 \mathrm{~Hz}$ the rate is $3.4 \mu \mathrm{m}$ per $100 \mu \mathrm{m}$ displacement range, and at $5 \mathrm{~Hz}$ it is $3.2 \mu \mathrm{m}$ per $100 \mu \mathrm{m}$ range as illustrated in Fig. 8(d). From these results, we conclude that for a given displacement range, charge control yields significantly less hysteresis compared to voltage control. In fact, the results show that charge control can reduce the displacement hysteresis by over $80 \%$ when the range is $700 \mu m$ (see Fig. 7(c2) and Fig. 8(c2)).

Finally, we conclude by noting that as the frequency increases from $1 \mathrm{~Hz}$ to $5 \mathrm{~Hz}$, hysteresis is more sensitive to the change in frequency under voltage control. But it is not the case with charge control (e.g. , see Fig. 8(d)). For instance, with charge control at $1 \mathrm{~Hz}$, there is $3.4 \mu \mathrm{m}$ displacement hysteresis per $100 \mu \mathrm{m}$ displacement range, and at $5 \mathrm{~Hz}$ it is roughly the same at $3.2 \mu \mathrm{m}$ per $100 \mu \mathrm{m}$. With voltage control, the difference from $1 \mathrm{~Hz}$ to $5 \mathrm{~Hz}$ is nearly half. The charge controller's ability to correct for dynamic changes is related to the use of feedback mechanism, i.e., controlling the charge delivered to the piezo load by monitoring the voltage and the charge on the electrodes.

\section{CONCLUSIONS}

We studied the displacement behavior of a LIPCA device subjected to voltage and charge-feedback control. At relatively slow scan speed (1/60 Hz scan frequency), creep causes an increase in displacement hysteresis by at least 25\%. Experimental results comparing the effect of voltage and charge control demonstrate that charge control reduces displacement hysteresis by over $80 \%$ for a LIPCA device that operates over a $700 \mu m$ range.

\section{ACKNOWLEDGMENTS}

The authors would like to acknowledge Bill Maryniak and Trek Incorporated for their support of this project. 
(a1)

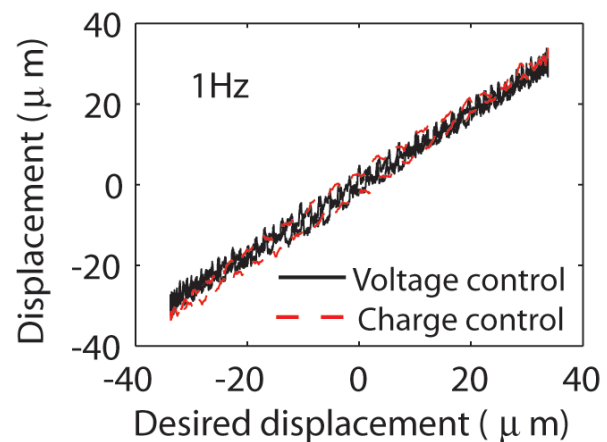

(b1)

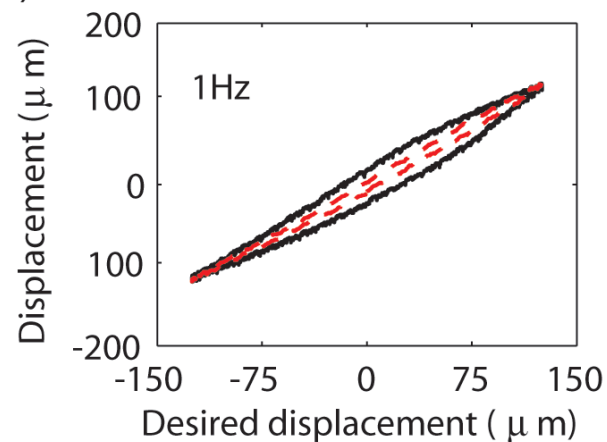

(c1)

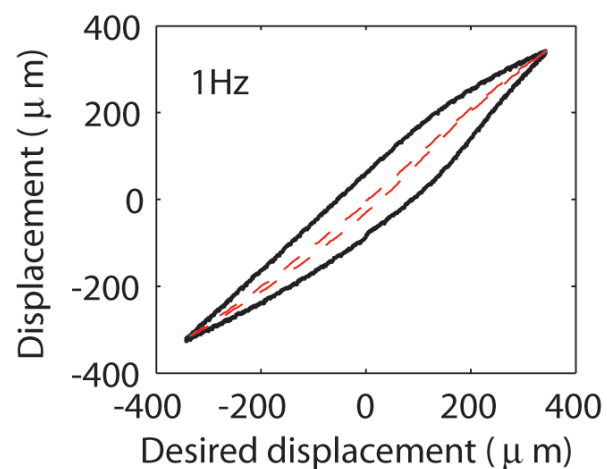

(a2)

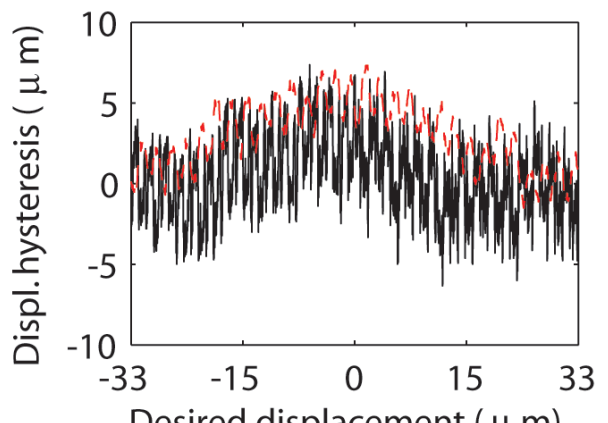

Desired displacement $(\mu \mathrm{m})$ (b2)

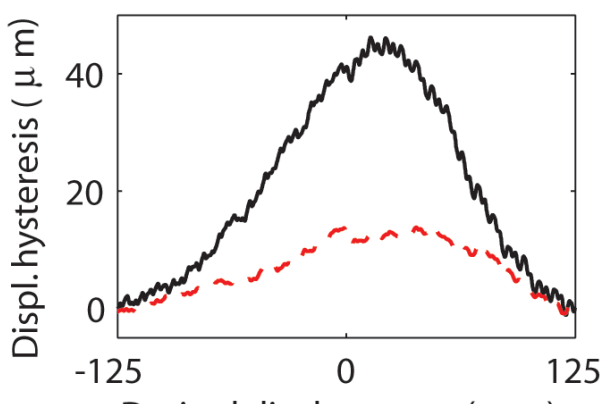

(c2)

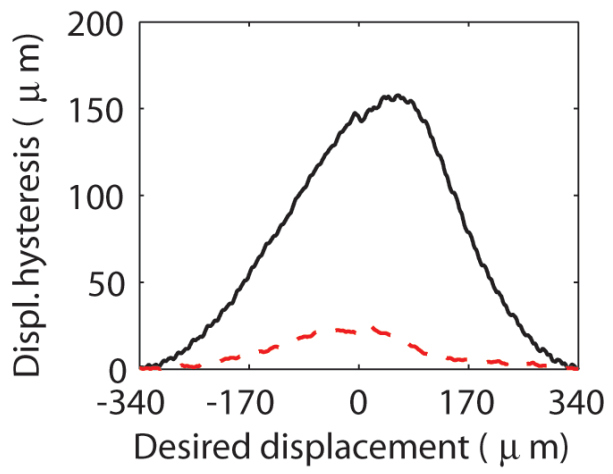

Figure 7. Hysteresis behavior for voltage and charge control at $1 \mathrm{~Hz}$ - solid and dashed lines represent voltage and charge control, respectively. (a) Small range, $70 \mu m( \pm 35 \mu m)$; (b) Medium range, $250 \mu m( \pm 125 \mu m)$; (c) Large range $700 \mu m( \pm 350 \mu m)$. 


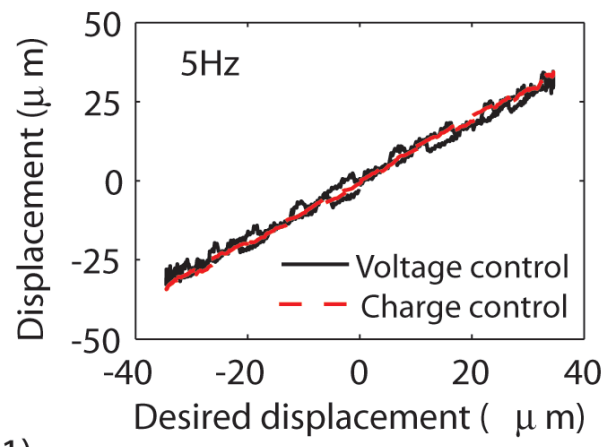

(b1)

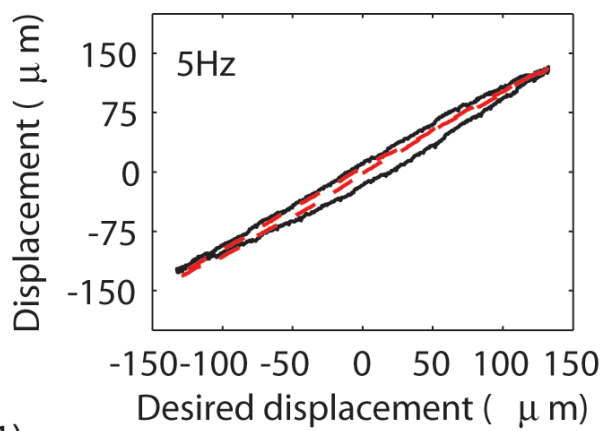

(c1)

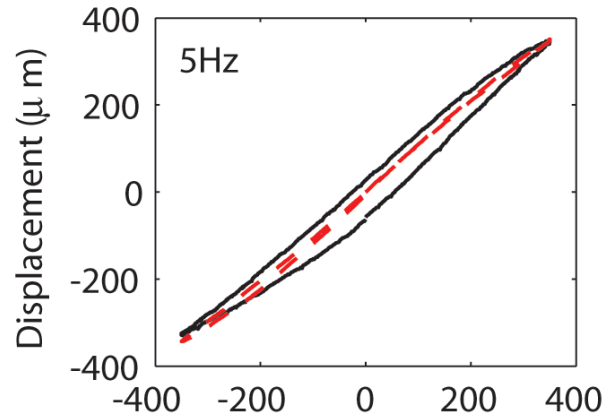

(d)

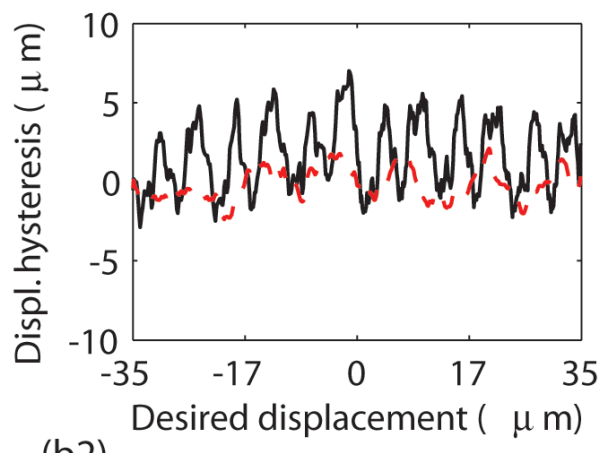

(b2)

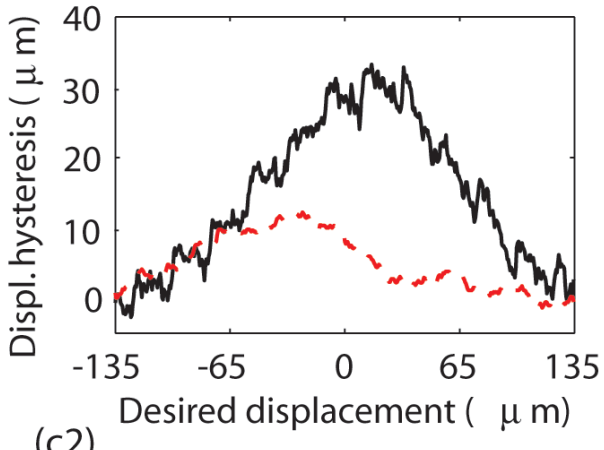

(c2)

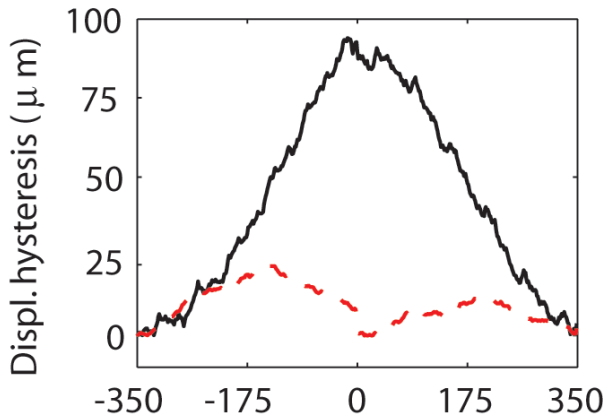

Desired displacement $(\mu \mathrm{m})$

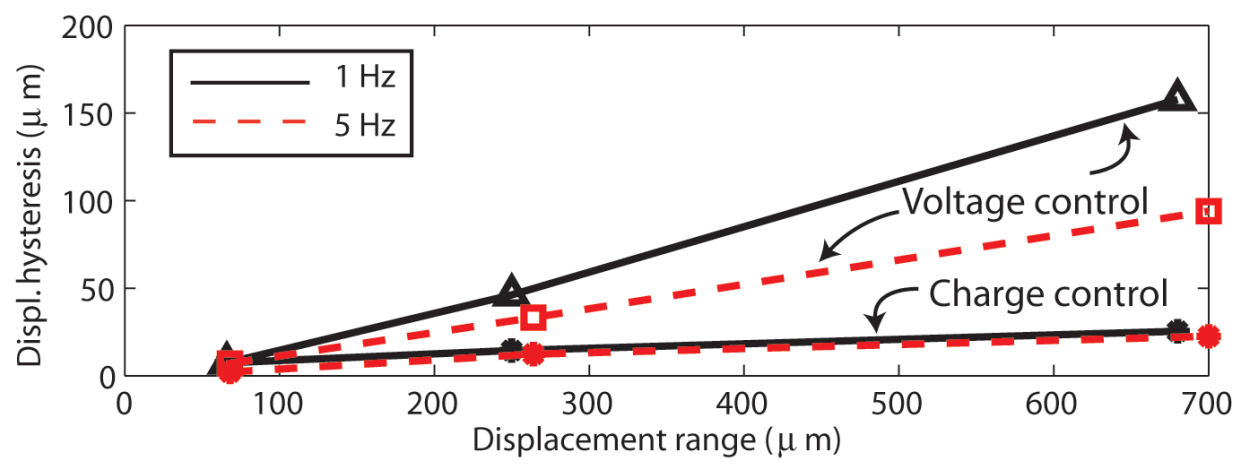

Figure 8. Hysteresis behavior for voltage and charge control at $5 \mathrm{~Hz}$. For (a) - (c), solid and dashed lines represent voltage and charge control, respectively. (a) Small range, $70 \mu \mathrm{m}( \pm 35 \mu \mathrm{m})$; (b) Medium range, $250 \mu \mathrm{m}( \pm 125 \mu \mathrm{m})$; (c) Large range $700 \mu m( \pm 350 \mu m)$; (d) Displacement hysteresis, $D_{h}(u)$, versus displacement range. 


\section{REFERENCES}

1. G. H. Haertling, "Rainbow ceramics - a new type of ultra-high-displacement actuator," Am. Ceram. Soc. Bulletin 73, pp. 93-96, 1994.

2. K. K. Mossi and R. Bryant, "Piezoelectric actuators for synthetic jet applications," in Materials Research Society Symposium - Proceedings on Materials and Devices for Smart Systems, $\mathbf{7 8 5}$.

3. A. Haris, N. S. Goo, H. C. Park, and K. J. Yoon, "Modeling and analysis for the development of Lightweight Piezoceramic Composite Actuators (LIPCA)," Computational Materials Science 30, pp. 474-481, 2004.

4. K. J. Yoon, S. Shin, H. C. Park, and N. S. Goo, "Design and manufacture of a lightweight piezo-composite curved actuator," Smart Mater. Struct. 11, pp. 163-168, 2002.

5. S. M. Lim, S. Lee, H. C. Park, and K. J. Yoon, "Design and demonstration of a biomimetic wing section using a lightweight piezo-composite actuator (LIPCA)," Smart Materials and Structures 14, pp. 495-503, 2005.

6. K. Mossi, Z. Ounaies, R. C. Smith, and B. Ball, "Pre-stressed curved actuators: characterization and modeling of their piezoelectric behaviors," Tech. Rep. CRSC-TR03-17, Center for Research in Scientific Computation, North Carolina State University, April, 2003.

7. K. Furutani, M. Urushibata, and N. Mohri, "Improvement of control method for piezoelectric actuator by combining induced charge feedback with inverse transfer function compensation," in Proc. IEEE Int. Conf. on Robotics and Automation, pp. 1504-1509, (Leuven, Belgium), May 1998.

8. G. Binnig, "Force microscopy," Ultramicroscopy 42-44, pp. 7-15, 1992.

9. J. A. Main and E. Garcia, "Piezoelectric stack actuators and control system design: strategies and pitfalls," AIAA J. Guidance, Control, and Dynamics 20, pp. 479-485, May-June 1997.

10. A. J. Fleming and S. O. R. Moheimani, "A grounded-load charge amplifier for reducing hysteresis in piezoelectric tube scanners," Review of Scientific Instruments 76, pp. 073707-1-073707-5, 2005.

11. C. Newcomb and I. Flinn, "Improving the linearity of piezoelectric ceramic actuators," Elec. Let. 18, pp. 442 444, 1982.

12. K. Furutani, M. Urushibata, and N. Mohri, "Displacement control of piezoelectric element by feedback of induced charge," Nanotechnology 9, pp. 93-98, 1998.

13. S. O. R. Moheimani and B. J. G. Vautier, "Resonant control of structural vibration using charge-driven piezoelectric actuators," IEEE Transaction on Control Systems Technology 13, pp. 1021-1035, November 2005.

14. R. Schwartz and M. Narayanan, "Development of high performance stress-biased actuators through the incorporation of mechanical pre-loads," Sensors and Actuators A 101, pp. 322-331, 2002.

15. J. A. Main, E. Garcia, and D. V. Netwon, "Precision position control of piezoelectric actuators using charge feedback," AIAA J. Guidance, Control, and Dynamics 18, pp. 1068-1073, September-October 1995.

16. H. Kaizuka, "Application of capacitor insertion method to scanning tunneling microscopes," Rev. Sci. Instr. 60, pp. 3119-3122, October 1989.

17. K. R. Koops, P. M. L. O. Scholte, and W. L. de Koning, "Observation of zero creep in piezoelectric actuators," Applied Physics A 68, pp. 691-697, 1999.

18. H. Jung and D.-G. Gweon, "Creep characteristics of piezoelectric actuators," Rev. Sci. Instr. 71, pp. 18961900, April 2000.

19. D. Croft, G. Shed, and S. Devasia, "Creep, hysteresis, and vibration compensation for piezoactuators: atomic force microscopy application," ASME J. Dyn. Syst., Meas., and Control 123, pp. 35-43, March 2001.

20. K. K. Leang and S. Devasia, "Design of hysteresis-compensating iterative learning control for piezopositioners: application to atomic force microscopes," Mechatronics, 2006. In Press.

21. L. Malvern, Introduction to the mechanics of a continuous medium, Prentice-Hall, Englewood Cliffs, NJ, 1969.

22. G. F. Franklin, J. D. Powell, and A. Emami-Naeini, Feedback control of dynamics systems, 3rd Edition, Addison-Wesley, New York, 1994. 